\title{
Response to olaparib in metastatic castration-resistant prostate cancer with germline BRCA2 mutation: a case report
}

\author{
Yi Ma', Lijie He ${ }^{1 *}$ D, Qianwen Huang ${ }^{2}$, Shuang Zheng ${ }^{1}$, Zhiqiang Zhang ${ }^{1}$, Hongshi Li ${ }^{1}$ and Shuang Liu
}

\begin{abstract}
Background: Prostate cancer is a heterogeneous disease, meaning patients would benefit from different treatment strategies based on their molecular stratification. In recent years, several genomic studies have identified prostate cancers with defects in DNA repair genes. It is known that the PARP inhibitor, olaparib, has a significant synthetic lethal effect on tumors with BRCA 1/2 mutations, particularly in ovarian and breast cancer.

Case presentation: In this study, we describe a patient with metastatic castration-resistant prostate cancer ( $\mathrm{mCRPC}$ ) containing a BRCA2 germline mutation who underwent olaparib treatment. The efficacy of the treatment was monitored by serum TPSA level as well as mutation levels of circulating tumor DNA (ctDNA) using nextgeneration sequencing (NGS). The patient responded to the olaparib treatment as indicated by the minimal residual levels of TPSA and tumor-specific mutations of ctDNA in plasma after four months of treatment, although the patient eventually progressed at six-month post-treatment with significantly elevated and newly acquired somatic mutations in ctDNA.
\end{abstract}

Conclusions: Our study provides evidence that MCRPC with BRCA2 germline mutations could response to PARP inhibitor, which improves patient's outcome. We further demonstrated that NGS-based genetic testing on liquid biopsy can be used to dynamically monitor the efficacy of treatment.

Keywords: Metastatic prostate cancer, BRCA2 germline mutation, PARP inhibitor, Olaparib, Liquid biopsy

\section{Background}

Germline BRCA1/2 mutations are the greatest risk factor for inheritable breast and ovarian cancer [1]. In contrast to the diverse functions of BRCA1 in multiple DNA repair pathways and in checkpoint regulation, BRCA2 is mainly anticipated in DNA double strand breaks (DSBs) repair through RAD51-dependent homologous recombination (HR) [2]. Deleterious mutations in BRCA2 was also implicated in a high risk of prostate cancer predisposition (8.6-fold in men $\leq 65$ years) and more aggressiveness, as well as BRCA1 mutations although with a much lower frequency [3-5].

Poly(ADP-ribose) polymerases (PARPs) are nuclear enzymes playing important roles in various cellular processes including DNA repair [6]. Tumor cells defective

\footnotetext{
*Correspondence: 17702488896@163.com

'Department of Medical Oncology, People's Hospital of Liaoning Province,

No.33, Wenyi Road, Shenhe District, Shenyang City 110000 , China

Full list of author information is available at the end of the article
}

in $B R C A 1 / 2$ may rely on PAPR-dependent DNA repair, and therefore are sensitive to PARP inhibitors, which may also increase the sensitivity of tumor cells to DNA-damaging agents. Olaparib, a PARP inhibitor, has been approved by the US Food and Drug Administration (FDA) and European Medicines Agency registration for treatment of breast and ovarian cancer associated with BRCA $1 / 2$ defects [7, 8]. Sustained responses to PARP inhibitors have also been reported in metastatic prostate cancers with DNA-repair gene mutation $[9,10]$. Here we report a patient with germline BRCA2-mutated metastatic castration-resistant prostate cancer (mCRPC) who responded to the PARP inhibitor, olaparib.

\section{Case presentation}

The patient was a 67-year-old man who presented with dysuria. Computed tomography (CT) examination of the upper abdomen revealed multiple swollen retroperitoneal and pelvic lymph nodes and abnormal bone density 
on January 5th 2015. Positron Emission Tomography-CT (PET-CT) revealed hypermetabolic lesions in the left lobe of the prostate, and multiple bone sites, as well as enlarged lymph nodes of the left neck, supraclavicular region, retroperitoneal, bilateral iliac blood vessels and pelvic left side wall, which were diagnosed as malignant metastasis on February 5th 2015. A prostate biopsy was performed on February 28th 2015, and histologic assessment showed conventional adenocarcinoma with Gleason score $4+3=$ 7 , while serum TPSA level was $>100 \mathrm{ng} / \mathrm{mL}$. The clinical course of the patient was summarized in Fig. 1.

The patient started the treatment with bicalutamide tablets, zoladex and zoledronate on February 28th 2015. His TPSA level dropped to $13 \mathrm{ng} / \mathrm{mL}$ after two months of treatment, and he continued on the therapy. However, recurrent disease developed on July 9th 2015, marked by elevated TPSA up to $60 \mathrm{ng} / \mathrm{mL}$. The patient was then switched to the treatment with flutamide and zoledronate. On October 10th 2015, due to persistent increase in TPSA level, the patient was further treated with abiraterone. On February 5th 2016, emission CT showed progression with bone metastases, with TPSA level rising to $150 \mathrm{ng} / \mathrm{mL}$. The patient then started six cycles of systemic chemotherapy with docetaxel and metacortandracin, during which time his TPSA level continued to rise. One month after finishing the systemic chemotherapy, his TPSA level reached $492.3 \mathrm{ng} / \mathrm{mL}$. The patient then received enzalutamide, but by August 22nd 2016, the TPSA level had risen to $644.3 \mathrm{ng} / \mathrm{mL}$.

Considering the poor responses to all currently available therapies, we performed genetic testing on patient's circulating tumor DNA (ctDNA) from blood using next-generation sequencing (NGS) targeting over 400 cancer-relevant genes. The assay was done using a commercial test. Genomic DNA from the whole blood sample was used as germline control. We detected several genomic alterations known to be associated with prostate cancer; specifically, we identified PIK3$C A-\mathrm{Q} 546 \mathrm{~K}$ activated mutation with a mutant allele frequency (MAF) of 17\%, a TP53-DISCIFP1 fusion (MAF: $12 \%), 4.1$ folds of relative copy number gain of the $A R$ gene, as well as germline BRCA2-G1761X mutation. As a result, the patient started treatment with everolimus, a mTOR inhibitor, for his high MAF of PIK3CA-Q546K mutation. Despite this however, serum TPSA continued to increase slowly $798.9 \mathrm{ng} / \mathrm{mL}$ to $1379 \mathrm{ng} / \mathrm{mL}$. On October 27th 2016, CT scan showed progression of multiple lymph nodes metastases, double pleural effusion and appearance of new liver metastases (Fig. 2a). The patient also developed a fever, shortness of breath and lethargy followed by unconsciousness. The patient was transferred to the intensive care unit (ICU) and underwent transfusion, respirator assisted ventilation and tracheotomy.

Due to prior detection of the BRCA2 G1761X germline mutation and poor physical condition, the patient started on olaparib treatment, $400 \mathrm{mg}$ twice daily by nasal feeding tube, on November 1st 2016; the patient tolerated the dose and his symptoms significantly relieved. On December 26th 2016, CT assessment indicated a partial response (PR) of liver metastases to olaparib (Fig. 2b). Furthermore, TPSA level was reduced from $1379 \mathrm{ng} / \mathrm{mL}$ to $208 \mathrm{ng} / \mathrm{mL}$. Following resolution of fever, shortness of breath, lethargy and unconsciousness, the patient was transferred out of the ICU. On January 22nd 2017, the patient's blood sample was obtained for ctDNA testing by NGS, which showed that the tumor specific mutations identified before the treatment had significantly decreased (PIK3CA-Q546K, 0.4\%; TP53-DISCIFP1 fusion, $0.1 \%$; undetectable copy number gain of $A R$ ). After

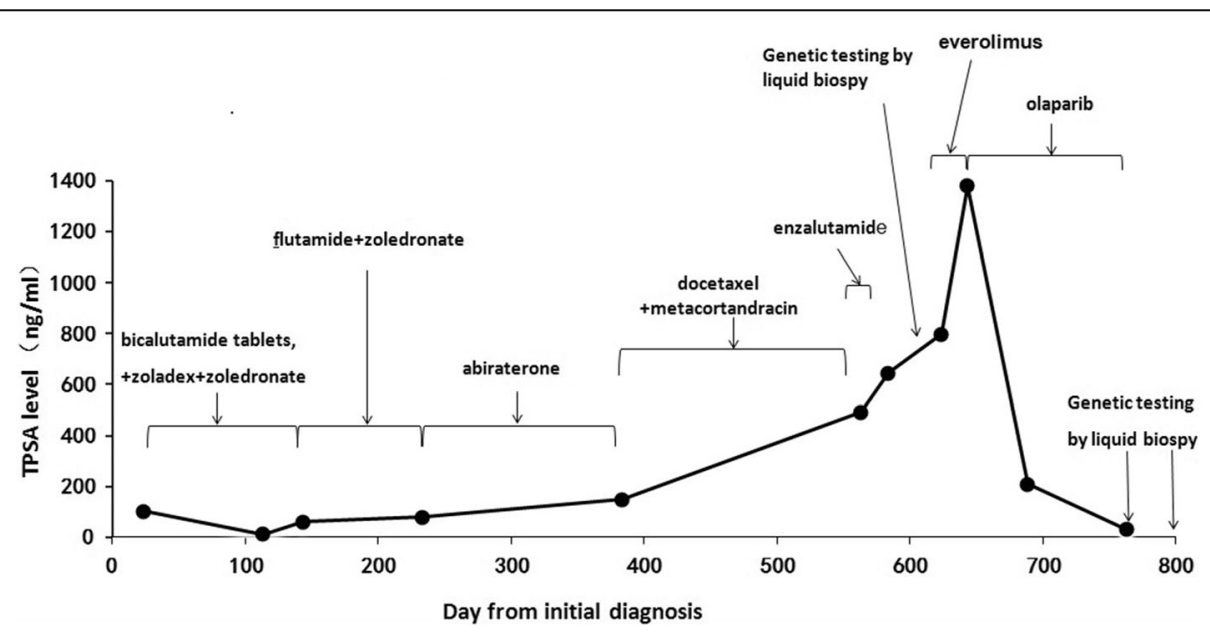

Fig. 1 Clinical course of the patient. Serum TPSA level was measured for disease monitoring. The timeline and duration of different treatments were indicated, as well as the time points for genetic testing 


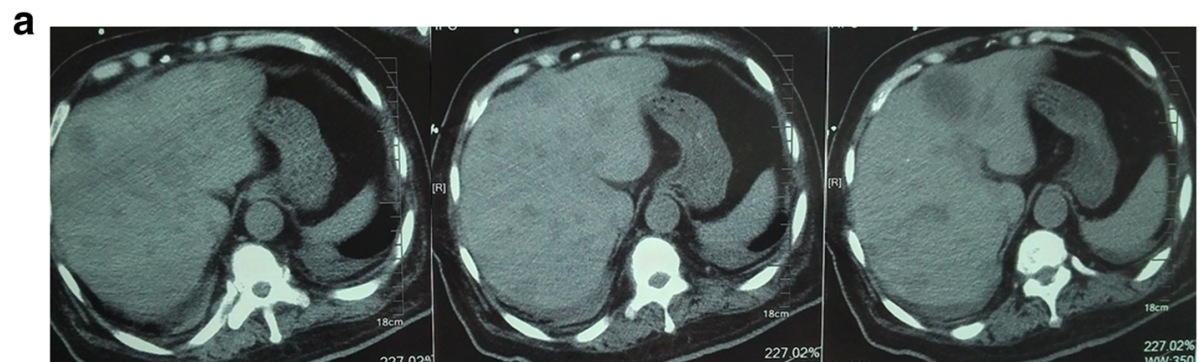

b

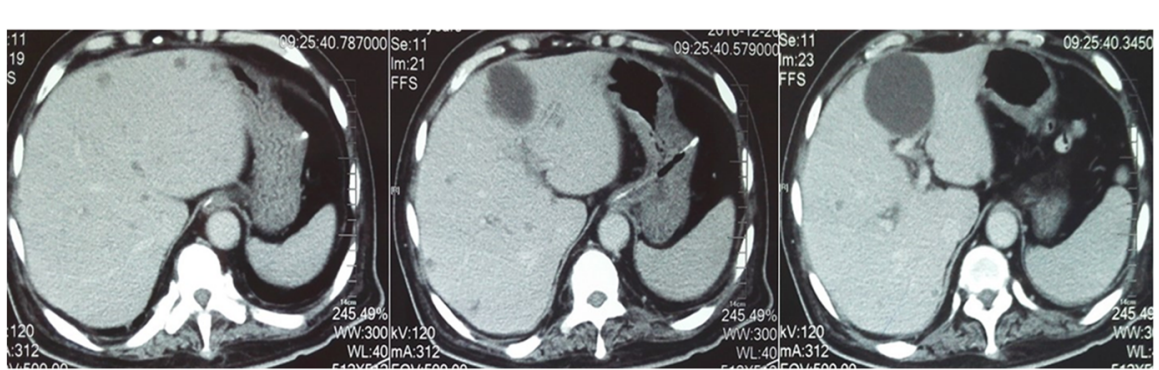

Fig. 2 Shrinkage of the patient's intra-hepatic lesions after two months of olaparib treatment. CT scan of the abdomen before (a) and after (b) two months of olaparib treatment

four months of the therapy, his TPSA level continued to fall to $30.65 \mathrm{ng} / \mathrm{mL}$. However, unfortunately, the patient's disease progressed again after six-month of the treatment, and his ctDNA testing showed that all the previous detected tumor specific mutations elevated to an even higher level compared to pretreatment (PIK3CA-Q546K, 19.9\%; TP53-DISCIFP1 fusion, 29.1\%; 4.1 folds of relative copy number gain of $A R$ ), as well as a newly emerged $R B 1$ single copy number loss. In addition, some other somatic genomic alterations had been found in the third test (Table 1).

\section{Discussion and conclusions}

PARP inhibitors have proven effective in patients with breast and ovarian cancers harboring BRCA1/2 mutations. Preliminary data also showed activity of these drugs in patients with germline BRCA1/2-mutated prostate cancer [7]. In this study, we observed a patient with germline BRCA2 G1761X mutation as well as somatic PIK3CA Q546K mutation, a TP53-DISCIFP1 fusion and $A R$ gene copy number gain, who had a favorable response to olaparib, although the patient eventually progressed with the emergence of olaparib resistance after six months of treatment. During the olaparib-response period, we found via liquid biopsy that the MAF of PIK3CA Q546K mutation decreased from 17 to $0.4 \%$, which then increased back to $19.9 \%$ upon patient's progression. NGS genetic testing further demonstrated that the MAF of TP53-DISCIFP1 fusion decreased from 12 to $0.1 \%$ in response to olaparib treatment, and then increased to $29.1 \%$ when the disease progressed.
Preclinical models have suggested that PIK3CA pathway activation can alter AR transcriptional activity and lead to hormonal therapy resistance [11, 12]. A recent publication suggest that patients has longer PFS with normal PIK3CA versus those with mutation or activation [13]. This patient had poor responses to all hormonal therapies. However, the role of PIK3CA mutations in olaparib susceptibility are not currently known. We need futher research.

A recent study suggesting that outcomes to abiraterone and enzalutamide appear better in MCRPC patients harboring germline BRCA/ATM mutations (vs no mutations), but not for patients with other non-BRCA/ATM germline mutations [14]. Another recent study suggesting that men with germline and/or somatic DNA repair gene alterations may have a better response to firstline abiraterone treatment (with or without concurrent use of a PARP inhibitor) than those without mutations. This study also suggesting that patients has longer PFS with normal PTEN, TP53, and PIK3CA versus those with mutation or activation.Futher multivariable analysis including clinical and biomarker variables individually revealed DRD(DNA-damage repair defect) and TP53 as biomarkers separately associated with PFS after controlling for clinical covariates [13]. Although this patient had germline DNA repair gene alterations (BRCA2), he did not had a good response to abiraterone and a PARP inhibitor. So we suppose that the TP53 alterations perhaps dominated the tumor biology in this case and not the BRCA2 lesion. The TP53 fusion is probably pathogenic, especially if it disrupts any of the key functional domains of the p53 protein. Studies on large case series demonstrate that TP53 





mutations are independent markers of bad prognosis in breast and several other cancers, and that the exact type and position of the mutation influences disease outcome [15].

In addition, when the patient's disease progressed after the treatment of olaparib, and the ctDNA testing showed that a newly emerged $R B 1$ single copy number loss. RB1 alteration is rare in primary prostate adenocarcinoma [16], unlike PTEN or TP53 mutation. Previous papers have suggested that Retinoblastoma (RB1) and tumor protein 53 (TP53) tumor suppressor gene loss drives transformation of prostate adenocarcinoma (PADC) to neuroendocrine prostate cancer variants (NEPC) resistant to antiandrogen therapy (AAT) [17]. This hypothesis potentially extends beyond prostate cancer since neuroendocrine lineage transformation associated with RB1 and TP53 loss has also been observed in lung adenocarcinoma relapsing from epidermal growth factor receptor-targeted therapies [18].That may also one of the mechanisms of PARP inhibitors resistance. We need further molecular based investigantionsto identify the hypothesis.

Approximately $20 \%$ of metastatic prostate cancers harbor mutations in genes required for DNA repair by homologous recombination (HRR) such as BRCA2. HRR defects confer synthetic lethality to PARP inhibitors (PARPi) such as olaparib [19].But tumors sensitive to PARP inhibitors are known to ultimately develop resistance, so far, multiple mechanisms have been proposed. First, olaparib can trigger secondary acquired $B R C A \mathrm{mu}-$ tations leading to restoration of the RAD51-dependent HR pathway and allow for doublestrand breaks to undergo this less destructive repair pathway [19-22]. Intriguingly, these reversion mutations can restore the open reading frame of HR genes (e.g. BRCA2, PALB2), these have been observed not only in the setting of somatic HR mutations but also apply to germline mutations. By reverting to wild-type, such cancer cells become HR-proficient meaning that they are no longer susceptible to synthetic lethality despite ongoing PARP inhibition [23]. This patient had a germline BRCA2 p.G1761X(c.G5281 T) mutation, at the time of progression, a further test was made, but we had neither found additional somatic BRCA2 mutations nor nucleotide sequences flanking the BRCA2 original frameshift deletions, so in this case, no ORF-restoring BRCA2 mutations (i.e. reversion mutations) were discovered on the progression ctDNA analysis. Second, Cells lacking HRR must repair double-strand DNA breaks through more error-prone forms of DNA repair such as non-homologous end joining which leads to worsening mutational burden [19]. The loss of a key regulatory protein within the non-homologous end junction repair pathway, 53BP1, promotes the increased utilization of HR [24]. If both of these deficits occur in concert, then partial ATM-dependent HR repair proceeds in BRCA1- but not
BRCA2-deficient cells [24, 25]. Of note, this escape mechanism has been identified clinically in BRCA1/2-associated breast cancer but may also mediate a proportion of prostate cancers that become resistant [25]. Third, upregulation of P-glycoprotein efflux transporter pumps reduces activity of many drugs, including PARP inhibitors, by depleting their intracellular availability [21,22].

We found several new somatic mutants (i.e. NKX2-1, ERBB4, RUNX1, NF1, MET, FGFR4 and TET2) when the disease progressed, now we did not know the correlation between the somatic mutants and the resistance, but compared with the second genetic test, more new mutants had appeared, which indicate that the tumor cells were in an extremely active state and need timely treatment. In addition, these aberrations again indicate possible divergent clonal evolutionary resistance mechanisms as a result of PARP inhibition-generated selective pressures [20]. Overall, this case demonstrates that the PARP inhibitor olaparib can be effective in treating patients with germline BRCA2 mutated prostate cancer and highlights the potential of NGS-based genetic testing on liquid biopsy as a diagnostic tool to monitor the presence and dynamics of tumor clones.

\section{Abbreviations \\ ctDNA: Circulating tumor DNA; FDA: US Food and Drug Administration; MAF: Mutant allele frequency; mCRPC: Metastatic castration-resistant prostate cancer; NGS: Next-generation sequencing; PARP: Poly-ADP-ribose polymerases; PET-CT: Positron Emission Tomography-CT}

\section{Acknowledgements}

We are grateful to the two reviewers for their comments that help significantly to improve the quality of this manuscript.

\section{Availability of data and materials}

The datasets generated and analysed during the current study are not publicly available due [my data relate to human research participants, it may not be appropriate to make them publicly available] but are available from the corresponding author on reasonable request.

\section{Authors' contributions}

$\mathrm{HL}$ and SL collected, analyzed and interpreted the data relative to the Case Report; SZ and ZZ performed the clinical assessment of the patient and wrote the manuscript; $\mathrm{QH}$ and YM conceived the work and critically revised the manuscript; LH given final approval of the version to be published. All authors have read and approved the final manuscript.

Ethics approval and consent to participate

The publication of this manuscript has been reviewed and approved by the People's Hospital of Liaoning Province institutional review board.

Consent for publication

The patient and his family have consent to publish the manuscript.

Competing interests

The authors declare that they have no competing interests.

\section{Publisher's Note}

Springer Nature remains neutral with regard to jurisdictional claims in published maps and institutional affiliations. 


\section{Author details}

'Department of Medical Oncology, People's Hospital of Liaoning Province, No.33, Wenyi Road, Shenhe District, Shenyang City 110000 , China. ${ }^{2}$ Dalian Medical University, Dalian 116000, China.

\section{Received: 12 April 2018 Accepted: 2 October 2018}

\section{Published online: 17 October 2018}

\section{References}

1. Chen S, Parmigiani G. Meta-analysis of BRCA1 and BRCA2 penetrance. J Clin Oncol. 2007:25(11):1329-33.

2. Roy R, Chun J, Powell SN. BRCA1 and BRCA2: different roles in a common pathway of genome protection. Nat Rev Cancer. 2011;12(1):68-78

3. Kote-Jarai Z, Leongamornlert D, Saunders E, Tymrakiewicz M, Castro E, Mahmud N, Guy M, Edwards S, O'Brien L, Sawyer E, et al. BRCA2 is a moderate penetrance gene contributing to young-onset prostate cancer: implications for genetic testing in prostate cancer patients. $\mathrm{Br} J$ Cancer. 2011;105(8):1230-4.

4. Tryggvadottir L, Vidarsdottir L, Thorgeirsson T, Jonasson JG, Olafsdottir EJ, Olafsdottir GH, Rafnar T, Thorlacius S, Jonsson E, Eyfjord JE, et al. Prostate cancer progression and survival in BRCA2 mutation carriers. J Natl Cancer Inst. 2007:99(12):929-35.

5. Narod SA, Neuhausen S, Vichodez G, Armel S, Lynch HT, Ghadirian P, Cummings S, Olopade O, Stoppa-Lyonnet D, Couch F, et al. Rapid progression of prostate cancer in men with a BRCA2 mutation. $\mathrm{Br} J$ Cancer. 2008:99(2):371-4.

6. Morales J, Li L, Fattah FJ, Dong Y, Bey EA, Patel M, Gao J, Boothman DA. Review of poly (ADP-ribose) polymerase (PARP) mechanisms of action and rationale for targeting in cancer and other diseases. Crit Rev Eukaryot Gene Expr. 2014;24(1):15-28.

7. Robson M, Im SA, Senkus E, Xu B, Domchek SM, Masuda N, Delaloge S, Li $W$, Tung N, Armstrong A, et al. Olaparib for metastatic breast Cancer in patients with a germline BRCA mutation. N Engl J Med. 2017;377(6):523-33.

8. Matulonis UA, Harter P, Gourley C, Friedlander M, Vergote I, Rustin G, Scott C, Meier W, Shapira-Frommer R, Safra T, et al. Olaparib maintenance therapy in patients with platinum-sensitive, relapsed serous ovarian cancer and a BRCA mutation: overall survival adjusted for postprogression poly(adenosine diphosphate ribose) polymerase inhibitor therapy. Cancer. 2016;122(12):1844-52.

9. Mateo J, Carreira S, Sandhu S, Miranda S, Mossop H, Perez-Lopez R, Nava Rodrigues D, Robinson D, Omlin A, Tunariu N, et al. DNA-repair defects and Olaparib in metastatic prostate Cancer. N Engl J Med. 2015; 373(18):1697-708

10. De Felice F, Tombolini V, Marampon F, Musella A, Marchetti C. Defective DNA repair mechanisms in prostate cancer: impact of olaparib. Drug Des Devel Ther. 2017;11:547-52

11. Jiao J, Wang S, Qiao R, et al. Murine cell linesderived fromPten null prostate cancer showthe critical role of PTEN in hormone refractory prostate cancer development. Cancer Res. 2007;67:6083-91.

12. Carver BS, Chapinski C, Wongvipat J, et al. Reciprocal feedback regulation of PI3K and androgen receptor signaling in PTEN-deficient prostate cancer. Cancer Cell. 2011;19:575-86.

13. Hussain M, Daignault-Newton S, Twardowski PW, et al. Targeting androgen receptor and DNA repair in metastatic castration-resistant prostate cancer: results from NCl 9012. J Clin Oncol. 2018:36:991-9.

14. Antonarakis ES, Lu C, et al. Germline DNA-repair gene mutations and outcomes in men with metastatic castration-resistant prostate cancer receiving first-line abiraterone and enzalutamide. Eur Urol. 2018;74:218-25.

15. Petitjean A, Achatz Ml, et al. TP53 mutations in human cancers: functional selection and impact on cancer prognosis and outcomes. Oncogene. 2007; 26(15):2157-65.

16. Schlomm T, et al. The molecular taxonomy of primary prostate cancer. Cell. 2015:163(4):1011-25

17. Wadosky KM, Ellis $L$, et al. Evasion of targeted cancer therapy through stemcell-like reprogramming. Mol Cell Oncol. 2017;4(2):2372-3556.

18. Niederst MJ, Sequist LV, Poirier JT, Mermel $\mathrm{CH}$, et al. Rb loss in resistant EGFR mutant lung adenocarcinomas that transform to small-cell lung cancer. Nat Commun. 2015;6:6377.

19. Quigley D, et al. Analysis of circulating cell-free DNA identifies multiclonal heterogeneity of BRCA2 reversion mutations associated with resistance to PARP inhibitors. Cancer Discov. 2017;7(9):999-1005.
20. Goodall J, et al. Circulating cell-free DNA to guide prostate cancer treatment with PARP inhibition. Cancer Discov. 2017;7(9):1006-17.

21. Lord $\mathrm{CJ}$, Ashworth $\mathrm{A}$, et al. Mechanisms of resistance to therapies targeting BRCA-mutant cancers. Nat Med. 2013;19(11):1381-8.

22. Tangutoori S, Baldwin P, Sridhar $S$, et al. PARP inhibitors: a new era of targeted therapy. Maturitas. 2015;81(1):5-9.

23. Christenson ES, Antonarakis ES, et al. PARP inhibitors for homologous recombination deficient prostate cancer. Expert Opin Emerg Drugs. 2018:23:123-33.

24. Montoni A, Robu M, Pouliot E, Shah GM, et al. Resistance to PARP-inhibitors in cancer therapy. Front Pharmacol. 2013;4:18.

25. Bouwman P, et al. 53BP1 loss rescues BRCA1 deficiency and is associated with triple-negative and BRCA-mutated breast cancers. Nat Struct Mol Biol. 2010;17(6):688-95
Ready to submit your research? Choose BMC and benefit from:

- fast, convenient online submission

- thorough peer review by experienced researchers in your field

- rapid publication on acceptance

- support for research data, including large and complex data types

- gold Open Access which fosters wider collaboration and increased citations

- maximum visibility for your research: over $100 \mathrm{M}$ website views per year

At $\mathrm{BMC}$, research is always in progress.

Learn more biomedcentral.com/submissions 